\title{
Evaluating the Gaia Contribution to the Field of Eclipsing Binaries with Ground-Based Spectroscopy and Hipparcos Photometry
}

\author{
P. G. Niarchos ${ }^{1} \dagger$, U. Munari ${ }^{2,3}$ and T. Zwitter ${ }^{4}$ \\ ${ }^{1}$ Department of Astrophysics, Astronomy and Mechanics, National and Kapodistrian \\ University of Athens, Athens, Greece \\ email: pniarcho@phys.uoa.gr \\ ${ }^{2}$ Osservatorio Astronomico di Padova, Sede di Asiago, 36012 Asiago (VI), Italy \\ ${ }^{3}$ Dipartimento di Astronomia dell'Universita di Padova, Osservatorio Astrofisico, 36012 Asiago \\ (VI), Italy \\ ${ }^{4}$ University of Ljubljana, Department of Physics, Jadranska 19, 1000 Ljubljana, Slovenia
}

\begin{abstract}
During its definition phase, ESA's Cornerstone mission Gaia was designed to perform extremely accurate photometry in 10 medium plus 5 broad bands and to collect about 90 epoch spectra with a resolving power of 11500 and a wavelength range $8480-8740 \AA$ (centered on the CaII triplet in the far red). Combining epoch photometry from the ground and the Hipparcos mission with ground-based spectra strictly simulating Gaia ones, we have investigated in a series of papers the performance expected from Gaia on SB2 EBs. We review here the results we obtained. The Gaia design is now under major revision, and its impact on EBs will be briefly addressed.
\end{abstract}

Keywords. Surveys - stars: fundamental parameters - binaries: eclipsing - binaries: spectroscopic

\section{Introduction}

Gaia is one of the next cornerstones of ESA's science programme (2000). It will be launched around end-2011 with a Soyuz-Fregat launcher and it will be put in a Lissajoustype orbit around the Sun-Earth Lagrangian point L2. The objectives of the Gaia mission are to build a catalogue of $\sim 10^{9}$ stars with accurate positions, parallaxes, proper motions, magnitudes and radial velocities. The catalogue will be complete up to $V=20^{\text {th }}$ mag with no input catalogue and therefore no associated bias.

The primary goal of Gaia is to explore the composition, formation and evolution of the Galaxy by studying the dynamics and intrinsic properties of a wide range of stellar types across the whole Galaxy. The quantities complementary to the kinematics can be derived from the spectral energy distribution of the stars by spectral photometry (dispersed images) and spectroscopy. The Gaia core science case requires measurement of: distance, space velocity, spectral energy distribution, duplicity, atmospheric parameters from which absolute quantities can be derived, like: luminosity, temperature, metallicity, galactic orbit.

The spacecraft and payload configuration was re-optimised by the industrial teams in their Phase B2/C/D proposal in response to the mission requirements document issued

$\dagger$ Present address: Dept. of Astrophysics, Astronomy and Mechanics, University of Athens, Greece. 
by the ESA project team in 2005. As a result, from early 2006, the final Gaia payload looks somewhat different from the previous design, although all functionality is preserved.

\section{Gaia's contribution to the study of eclipsing binaries}

Gaia is designed to obtain for a large sample of stars extremely precise micro-arcsec astrometry, spectral photometry with two photometers (Blue and Red) providing low resolution spectrophotometric measurements for each object over the wavelength ranges 330-660 and 650-1000 nm, respectively, and medium resolution spectroscopy. The RadialVelocity Spectrograph (RVS) will register spectra of all objects brighter than about $17^{\text {th }}$ mag.

It is expected that about $1 \times 10^{6} \mathrm{EBs}$ (with $V \leqslant 16^{\text {th }} \mathrm{mag}$ ) will be discovered and some $10^{5}$ of these will be characterized as double-lined in Gaia spectral observations. Moreover, most of the Gaia binaries will be of spectral type $\mathrm{G}$ or K (Zwitter \& Henden 2003) for which accurate solutions exist for only a small number of systems. Even if for only $1 \%$ of the observed EBs reliable parameters are derived, this will be a giant leap in comparison with what has been obtained so far from ground-based observations. The number of photometric points per star in the five-year mission lifetime is estimated to be $\sim 70$ to 100 and the number of spectra $\sim 40$. The observing fashion will be quite similar to Hipparcos operational mode.

The aim of the present investigation is to review the results of the investigations made so far aiming to evaluate the Gaia contribution to the field of Eclipsing Binaries. Such investigations for some detached, semi-detached and contact binaries of the basic spectral types A, F, G, K have been done by Munari et al. (2001), Niarchos \& Manimanis (2003), Zwitter et al. (2003), Marrese et al. (2004), Niarchos et al. (2005) and Milone et al. (2005). These investigations are based on ground-based and Hipparcos (Gaia-like) photometry, and on ground-based (Gaia-like) spectroscopy.

The first step for such an evaluation can be accomplished by studying a small sample of EBs, using ground-based and Hipparcos (Gaia-like) observations.

\section{Selection of systems}

There are crucial questions regarding the reliability of the derived stellar parameters from Gaia observations. Some of them are:

- How Gaia observations can be compared with the state-of-the-art ground-based observations?

- Can Gaia observations permit us to derive fundamental parameters as well or better than previous work?

- Can it add substantially to the list of very well-determined systems from groundbased data?

- What is the accuracy to which Close Eclipsing Binaries can be investigated using Gaia data alone?

The first step in our study was the selection of proper eclipsing systems to be analysed. The following criteria of selection were adopted:

(a) EBs observed by the Hipparcos/Tycho mission ( $H_{P}$ observations)

(b) Ground-based photometric observations of high quality for the same systems $(V$ observations)

(c) Accurate spectroscopic mass ratios for the same systems (determined from radialvelocity measurements using modern techniques) 
Table 1. Basic data of the systems of group 1

\begin{tabular}{lcccc}
\hline System & State & Spectral type & Mass ratio q & Reference \\
\hline RZ Dra & semi-detached & A5 + K2 & 0.444 & 1 \\
V1010 Oph & semi-detached & A5 + F6 & 0.448 & 1 \\
AB And & contact & G5 & 0.560 & 1 \\
$\epsilon$ CrA & contact & F2 & 0.112 & 1 \\
YY CrB & contact & F8 & 0.243 & 2 \\
XY Leo & contact & K0 & 0.500 & 2 \\
XZ Leo & contact & A5 & 0.348 & 2 \\
V566 Oph & contact & F4 + F8 & 0.237 & 2 \\
V839 Oph & contact & G0 & 0.305 & 2 \\
AH Vir & contact & K0 & 0.303 & 2 \\
\hline
\end{tabular}

1: Niarchos \& Manimanis 2003 (and references therein); 2: Niarchos et al. 2005 (and references therein).

Table 2. Basic data of the systems of group 2

\begin{tabular}{lcccc}
\hline System & State & Spectral type & Mass ratio q & Reference \\
\hline V432 Aur & detached & G0 & 0.560 & 3 \\
SV Cam & semi-detached & G5 & 0.303 & 4 \\
BS Dra & detached & F5 & 0.500 & 4 \\
GK Dra & detached & G0 & 0.112 & 2 \\
HP Dra & detached & G5 & 0.500 & 4 \\
UV Leo & detached & G0 & 0.237 & 2 \\
UW LMi & detached & G0 & 0.305 & 3 \\
CN Lyn & detached & F5 & 0.500 & 3 \\
OO Peg & detached & A2 & 0.348 & 1 \\
V505 Per & detached & F5 & 0.448 & 1 \\
V570 Per & detached & F5 & 0.444 & 1 \\
V781 Tau & contact & G0 & 0.243 & 2 \\
\hline
\end{tabular}

1: Munari et al. 2001 (and references therein); 2: Zwitter et al. 2003 (and references therein); 3: Marrese et al. 2004 (and references therein); 4: Milone et al. 2005 (and references therein).

(d) Main types of EBs (Detached, Semi-detached, Contact)

The systems selected were divided into two groups according to the observations used for their analysis: For the systems of group 1: $H_{P}$ photometry, ground-based $V$ photometry and accurate mass ratios determined fron ground-based spectroscopy were used; For the systems of group 2: $H_{P}$ photometry and accurate mass-ratios determined from ground-based Gaia-like spectroscopy (Asiago spectroscopy) were used. For one system of group 2 (V 505 Per) ground-based photometry was also used.

A higher resolving power $\mathrm{R}=\lambda / \Delta \lambda=17000$ for ground-based spectroscopy over the Gaia wavelength interval (8480-8740 $\AA$ ) has been adopted to compensate for the lower number of spectra that we have secured compared to the twice larger that Gaia will collect at a resolving power 11500. The spectroscopic observations have been obtained with the Echelle+CCD spectrograph on the 1.82-m telescope operated by Osservatorio Astronomico di Padova atop Mt. Ekar (Asiago). A $2^{\prime \prime} .2$ slit width was adopted to match the $\mathrm{R}=\lambda / \Delta \lambda=17000$ requirement. The detector was a UV-coated Thompson CCD $1024 \times 1024$ pixel, 19 micron square size. The Gaia spectral range is covered without gaps in a single order by the Asiago Echelle spectrograph. The actual observations however extended over a much larger wavelength interval (4500-9000 ̊). Here we will limit the analysis to the Gaia spectral interval. 

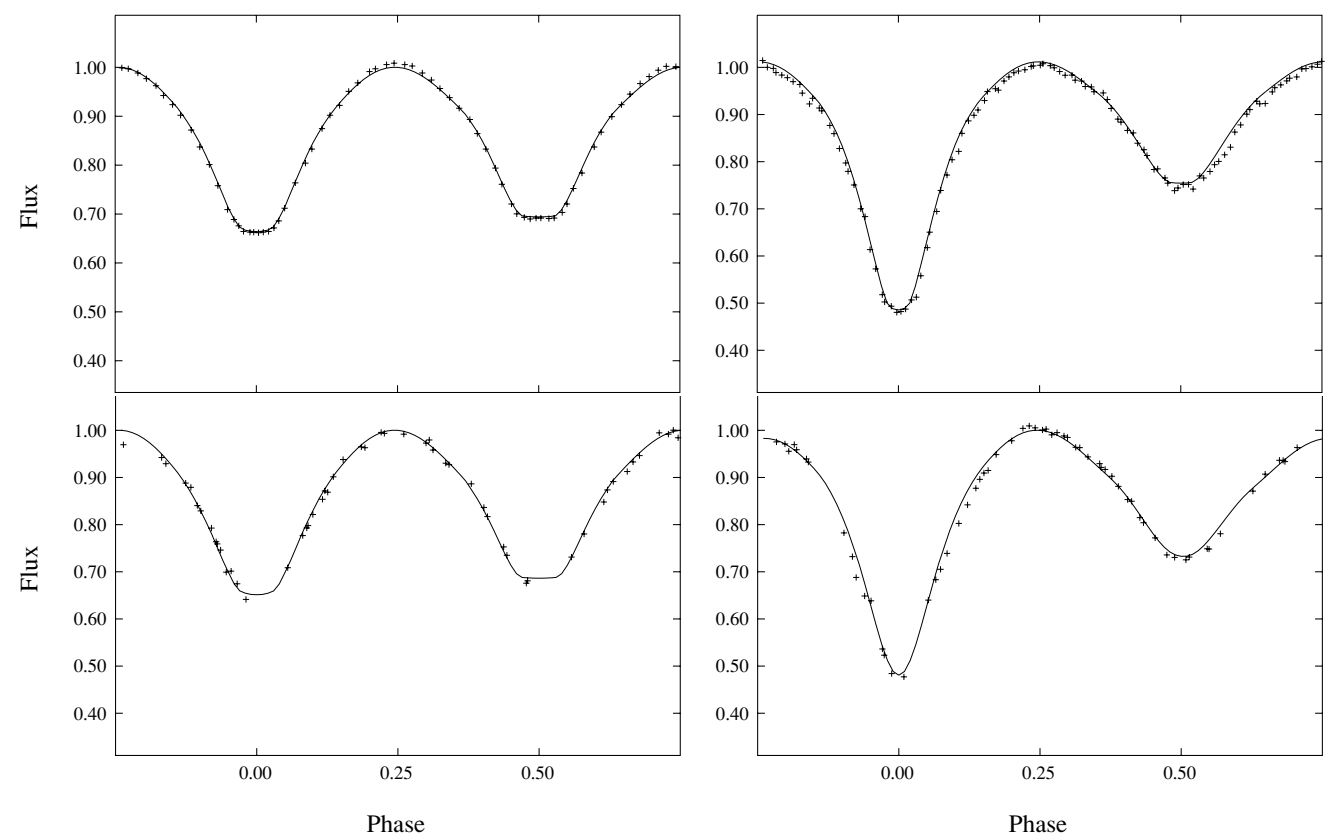

Figure 1. Light curves of the contact system V566 Oph (left) and the semi-detached system V1010 Oph (right). The upper curves are from ground-based $V$ observations and the lower ones from Hipparcos $H_{P}$ data. The solid lines represent our light-curve solutions.

\section{Method of analysis and comparison of absolute elements}

The Wilson-Devinney DC program was used for the analysis in the appropriate mode. The free parameters were: $\phi_{\circ}, i, T_{2}, \Omega$ (according to the mode used) and $L_{1}$, while the fixed ones were: $T_{1}$ (from spectral type); theoretical values for $g, A$ and $x$ were assumed based on spectral type. The spectroscopic mass ratio $q$ was used as fixed parameter for systems of group 1, while simultaneous radial velocity and light curve analysis was performed for systems of group 2. The third light was assumed zero in all cases.

The elements derived from the light-curve analyses were combined with the available spectroscopic elements in order to compute the absolute elements (physical parameters) of the systems studied. These parameters are listed in Tables 3 and 4 together with their percentage difference. Four examples of light curves and their solutions are given in Figures 1 and 2.

\section{Summary and Conclusions}

The agreement between the fundamental stellar parameters, derived from groundbased and Hipparcos (Gaia-like) observations is more than satisfactory. Their difference is within the limits of the combined errors. The stellar parameters could be determined (in many cases) at about $2 \%$ accuracy level. Of course there are exceptions for active systems and for those with poor photometric phase coverage.

The strength of the Gaia mission is in the numbers. Gaia will observe $\sim 4 \times 10^{5}$ eclipsing binaries brighter than $V \leqslant 15$ and $\sim 10^{5}$ of these will be double-lined (SB2) systems. For $V \leqslant 13$ the number of SB2 will be about 16000 for which Gaia should provide orbital solutions formally accurate to $\sim 2 \%$ (Munari et al. 2004). This is a fantastic number compared to $\leqslant 100$ systems studied at similar accuracy by ground-based observations so far. Moreover most of the Gaia binaries will be of G-K spectral type (Zwitter \& Henden 
Table 3. Absolute elements of group 2 systems in solar units

\begin{tabular}{lcccccc}
\hline System & $M_{1}$ & $M_{2}$ & $R_{1}$ & $R_{2}$ & $L_{1}$ & $L_{2}$ \\
\hline V432 Aur(2) & $0.98(2)$ & $1.06(2)$ & $1.39(8)$ & $2.13(14)$ & $2.36(20)$ & $4.86(35)$ \\
SV Cam(2) & $0.86(10)$ & $0.65(8)$ & $0.98(10)$ & $1.18(12)$ & $0.99(14)$ & $0.33(10)$ \\
BS Dra(2) & $1.29(8)$ & $1.28(9)$ & $1.46(2)$ & $1.40(7)$ & $3.61(4)$ & $3.34(18)$ \\
GK Dra(2) & $1.46(7)$ & $1.81(7)$ & $2.43(4)$ & $2.83(5)$ & $13.26(15)$ & $15.84(18)$ \\
HP Dra(2) & $1.10(2)$ & $1.10(3)$ & $1.17(3)$ & $0.89(9)$ & $1.57(5)$ & $1.16(16)$ \\
UV Leo(2) & $1.21(9)$ & $1.11(10)$ & $0.97(2)$ & $1.22(4)$ & $1.17(4)$ & $1.43(7)$ \\
UW LMi(2) & $1.06(2)$ & $1.04(2)$ & $1.23(5)$ & $1.21(6)$ & $2.39(16)$ & $2.31(7)$ \\
CN Lyn(2) & $1.04(2)$ & $1.04(2)$ & $1.80(21)$ & $1.84(24)$ & $5.11(53)$ & $5.19(60)$ \\
OO Peg(2) & $1.72(3)$ & $1.69(3)$ & $2.19(8)$ & $1.37(5)$ & $25.07(44)$ & $9.43(24)$ \\
\hline V505 Per(1) & $1.26(1)$ & $1.25(1)$ & $1.29(3)$ & $1.27(7)$ & $2.60(8)$ & $2.36(15)$ \\
V505 (2) & $1.30(2)$ & $1.28(2)$ & $1.40(2)$ & $1.14(3)$ & $2.96(5)$ & $1.94(6)$ \\
\% difference & $\mathbf{3 . 1}$ & $\mathbf{2 . 3}$ & $\mathbf{7 . 8}$ & $\mathbf{1 1 . 3}$ & $\mathbf{1 2 . 1}$ & $\mathbf{2 1 . 6}$ \\
\hline V570 Per & $1.28(3)$ & $1.22(3)$ & $1.64(16)$ & $1.01(25)$ & $4.14(37)$ & $1.33(41)$ \\
V781 Tau(2) & $0.51(1)$ & $1.15(3)$ & $0.76(1)$ & $1.11(1)$ & $0.85(2)$ & $1.57(2)$ \\
\hline
\end{tabular}

(1): ground-based observations (Marschall et al. 1997), (2): Gaia (expected) obs. (Munari et al. 2001, Zwitter et al. 2003, Marrese et al. 2004, Milone et al. 2005)

Table 4. Absolute elements of group 1 systems in solar units

\begin{tabular}{lcccccc}
\hline System & $M_{1}$ & $M_{2}$ & $R_{1}$ & $R_{2}$ & $L_{1}$ & $L_{2}$ \\
\hline RZ Dra (1) & $1.40(4)$ & $0.62(3)$ & $1.62(1)$ & $1.12(1)$ & $10.1(4)$ & $1.01(6)$ \\
RZ Dra (2) & $1.42(5)$ & $0.59(4)$ & $1.55(1)$ & $1.11(1)$ & $9.60(47)$ & $0.86(5)$ \\
\% difference & $\mathbf{1 . 4}$ & $\mathbf{4 . 8}$ & $\mathbf{4 . 3}$ & $\mathbf{0 . 9}$ & $\mathbf{5}$ & $\mathbf{1 5}$ \\
V1010 Oph (1) & $1.87(12)$ & $0.90(5)$ & $2.08(6)$ & $1.48(8)$ & $10.7(7)$ & $3.47(35)$ \\
V1010 Oph (2) & $1.88(14)$ & $0.89(7)$ & $2.08(1)$ & $1.46(1)$ & $11.7(8)$ & $3.07(8)$ \\
\% difference & $\mathbf{0 . 5}$ & $\mathbf{1 . 1}$ & $\mathbf{0}$ & $\mathbf{1 . 4}$ & $\mathbf{9 . 3}$ & $\mathbf{1 2}$ \\
AB And (1) & $1.01(2)$ & $0.56(1)$ & $1.04(4)$ & $0.80(5)$ & $0.87(7)$ & $0.46(5)$ \\
AB And (2) & $1.00(9)$ & $0.55(5)$ & $1.06(4)$ & $0.83(5)$ & $0.91(7)$ & $0.45(5)$ \\
\% difference & $\mathbf{1 . 0}$ & $\mathbf{1 . 8}$ & $\mathbf{1 . 9}$ & $\mathbf{3 . 7}$ & $\mathbf{4 . 6}$ & $\mathbf{2 . 2}$ \\
$\epsilon$ CrA (1) & $1.75(4)$ & $0.21(2)$ & $2.20(3)$ & $0.80(1)$ & $11.1(1)$ & $1.08(1)$ \\
CrA (2) & $1.69(7)$ & $0.22(5)$ & $2.12(12)$ & $0.80(4)$ & $10.3(20)$ & $1.07(14)$ \\
\% difference & $\mathbf{3 . 4}$ & $\mathbf{4 . 8}$ & $\mathbf{3 . 6}$ & $\mathbf{1 . 2}$ & $\mathbf{7 . 2}$ & $\mathbf{0 . 9}$ \\
YY CrB (1) & $1.41(9)$ & $0.34(2)$ & $1.40(7)$ & $0.77(10)$ & $2.46(26)$ & $0.81(20)$ \\
YY CrB (2) & $1.37(16)$ & $0.33(4)$ & $1.36(7)$ & $0.74(10)$ & $2.33(25)$ & $0.74(20)$ \\
\% difference & $\mathbf{2 . 8}$ & $\mathbf{2 . 9}$ & $\mathbf{2 . 9}$ & $\mathbf{3 . 9}$ & $\mathbf{5 . 3}$ & $\mathbf{8 . 6}$ \\
XY Leo (1) & $0.82(4)$ & $0.41(2)$ & $0.87(1)$ & $0.64(2)$ & $0.38(2)$ & $0.14(1)$ \\
XY Leo (2) & $0.83(21)$ & $0.41(10)$ & $0.86(1)$ & $0.62(2)$ & $0.37(2)$ & $0.17(1)$ \\
\% difference & $\mathbf{1 . 2}$ & $\mathbf{0 . 7}$ & $\mathbf{1 . 1}$ & $\mathbf{3 . 1}$ & $\mathbf{2 . 6}$ & $\mathbf{2 1 . 4}$ \\
XZ Leo (1) & $1.83(5)$ & $0.64(2)$ & $1.71(2)$ & $1.07(3)$ & $7.29(29)$ & $2.56(16)$ \\
XZ Leo (2) & $1.82(13)$ & $0.63(6)$ & $1.71(2)$ & $1.07(3)$ & $7.26(29)$ & $2.69(19)$ \\
\% difference & $\mathbf{0 . 5}$ & $\mathbf{1 . 6}$ & $\mathbf{0 . 2}$ & $\mathbf{0 . 2}$ & $\mathbf{0 . 4}$ & $\mathbf{5 . 1}$ \\
V566 Oph (1) & $1.40(3)$ & $0.33(1)$ & $1.47(1)$ & $0.79(1)$ & $4.57(4)$ & $1.26(1)$ \\
V566 Oph (2) & $1.54(11)$ & $0.36(4)$ & $1.518(3)$ & $0.819(4)$ & $4.99(24)$ & $1.26(2)$ \\
\% difference & $\mathbf{1 0}$ & $\mathbf{9 . 1}$ & $\mathbf{3 . 5}$ & $\mathbf{3 . 7}$ & $\mathbf{9 . 2}$ & $\mathbf{0 . 8}$ \\
V839 Oph (1) & $1.62(4)$ & $0.49(1)$ & $1.53(6)$ & $0.93(8)$ & $3.12(26)$ & $1.32(23)$ \\
V839 Oph (2) & $1.61(17)$ & $0.49(5)$ & $1.52(6)$ & $0.92(8)$ & $3.10(26)$ & $1.22(21)$ \\
\% difference & $\mathbf{0 . 6}$ & $\mathbf{0 . 8}$ & $\mathbf{0 . 7}$ & $\mathbf{1 . 1}$ & $\mathbf{0 . 6}$ & $\mathbf{7 . 6}$ \\
AH Vir (1) & $1.45(13)$ & $0.44(4)$ & $1.36(1)$ & $0.77(1)$ & $2.45(8)$ & $0.71(2)$ \\
AH Vir (2) & $1.33(20)$ & $0.40(6)$ & $1.44(1)$ & $0.88(1)$ & $2.76(9)$ & $0.91(3)$ \\
\% difference & $\mathbf{8 . 3}$ & $\mathbf{9 . 1}$ & $\mathbf{5 . 9}$ & $\mathbf{1 4 . 3}$ & $\mathbf{1 2 . 7}$ & $\mathbf{2 8 . 2}$ \\
\hline & & & & & & \\
\hline & & & & &
\end{tabular}

(1): ground-based observations, (2): Gaia (expected) observations (Niarchos \& Manimanis 2003, Niarchos et al. 2005) 

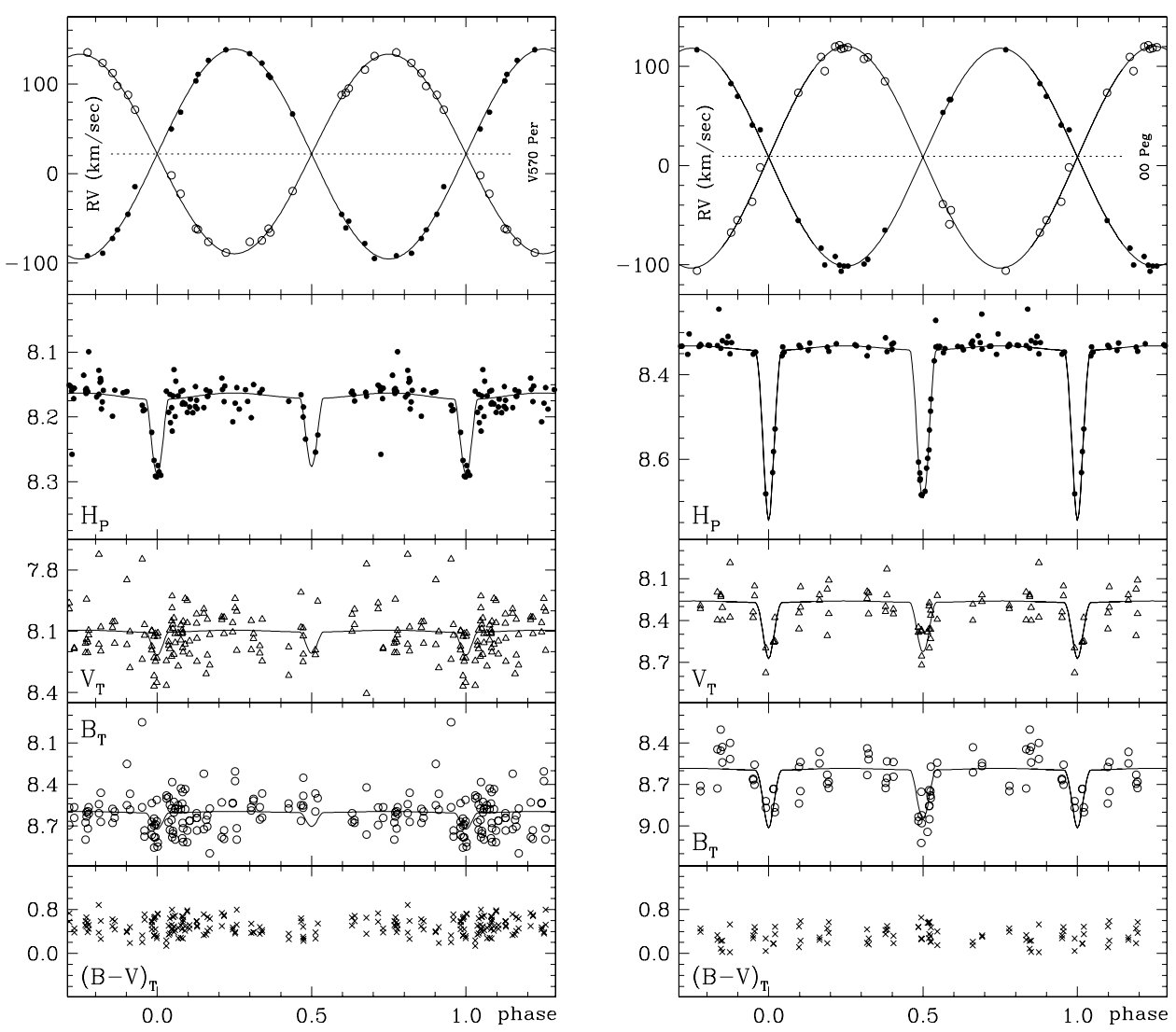

Figure 2. Radial velocity curves (from Asiago spectroscopy) and Hipparcos $H_{P}$ and Tycho $V_{T}$, $B_{T},(B-V)_{T}$ light curves of the detached systems V505 Per (left) and OO Peg (right). The solid lines represent our light curve solutions. (From Munari et al. 2001)

2003) for which accurate solutions exist only for a small number of systems. Although the expected accuracy will be moderate, the large amount of data will allow us to look for large deviations from the "normal" mass-radius-luminosity relations. There is no doubt that Gaia observations of EBs will have an immense impact on theories of stellar structure and evolution.

\section{References}

Marrese, P. M., Munari, U., Siviero, A., et al. 2004, A\&GA 413, 635 (Paper III)

Marschall, L. A., Stefanik, R. P., Lacy, C. H., et al. 1997, AJ 114, 793

Milone, E. F., Munari, U., Marrese, P. M., et al. 2005, A $₫ A$ 441, 605 (Paper IV)

Munari, U., Tomov, T., Zwitter, T. et al. 2001, A\&\&A 378, 477 (Paper I)

Munari, U., Zwitter, T., \& Milone, E. F. 2004, in: Hilditch, R., Hensberge, H. \& Pavlovski, K. (ed.) Spectroscopically and Spatially Resolving the Components of the Close Binary Stars (ASP Conf. Ser. 318), p. 422

Niarchos, P. \& Manimanis, V. 2003, A\& A 405, 263

Niarchos, P., Manimanis, V., \& Gazeas, K. 2005, in: Turon, C., O'Flaherty, K.S., \& Perryman, M.A.C. (ed.), The Three-Dimensional Universe with GAIA (ESA SP-576), p. 607

Zwitter, T. \& Henden, A.A. 2003, in: Munari, U. (ed.) Gaia Spectroscopy, Science and Technology (ASP Conf. Ser. 298), p. 489

Zwitter, T., Munari, U., Marrese, P. M., et al. 2003, AESA 404, 333 (Paper II) 